:::: Helmholtz-Zentrum

$\because \because:$ Geesthacht

Zentrum für Material- und Küstenforschung

\title{
Original
}

Schnubel, D.; Horstmann, M.; Huber, N.:

Retardation of fatigue crack growth in aircraft aluminium alloys via laser heating - Simulation based design optimization

In: International Journal of Structural Integrity (2013) Emerald Group

DOI: 10.1108/IJSI-08-2012-0020 


\section{Retardation of fatigue crack growth in aircraft aluminium alloys via laser heating Simulation-based design optimisation}

\author{
Dirk Schnubel, Manfred Horstmann and Norbert Huber \\ Helmholtz-Zentrum Geesthacht, Institute of Materials Research, \\ Materials Mechanics, Geesthacht, Germany
}

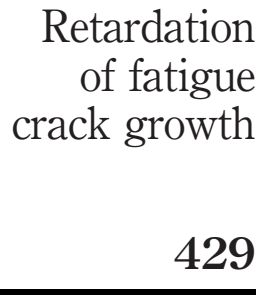

Received 30 August 2012 Revised 12 November 2012 Accepted 29 November 2012

\begin{abstract}
Purpose - While normally the formation of thermally induced residual stresses is seen mainly as detrimental side effect from production processes like welding or casting, the well-directed introduction of thermal residual stresses can also be used as tool to retard fatigue crack growth (FCG). In the presented paper, the use of a defocused laser to modify the residual stress state, and by that to retard the FCG, is examined. The focus lies on the simulation-based optimisation of the heating line position for achieving a maximum fatigue life. The paper aims to discuss these issues.

Design/methodology/approach - In the presented work, the developed prediction methodology for the FCG coupling process simulation and subsequent fracture mechanics analysis is used to identify the optimum positioning of either one or two heating lines on a C(T)100 specimen that leads to a maximised total lifetime. Afterwards, the prediction results are validated experimentally for selected cases.

Findings - The predictions match the experiments within the experimental scatter indicating the correct identification of the optimum heating line positions. This demonstrates the large potential for reducing the experimental effort needed for design optimisation using the proposed strategy.

Originality/value - The used methodology of coupling of welding simulation with subsequent fracture mechanics analysis in order to optimise the FCG behaviour of structures is innovative and only very few published studies addressed parts of similar approaches.
\end{abstract}

Keywords Fatigue crack growth, Laser heating, Numerical design optimisation,

Numerical fracture mechanics, Residual stress, Welding simulation

Paper type Research paper

\section{Introduction}

A major future challenge for the aircraft industry will be the development of proper strategies for maintenance and life extension of the growing ageing aircraft fleet. In this context, residual stress based life enhancement approaches are believed to have a huge potential as cost efficient solutions (McClung, 2007; Ball and Lowry, 1998; Reid, 2003).

While some researchers examined the possibility to use the well directed introduction of thermal residual stresses as tool to retard the fatigue crack growth (FCG) in thin walled structures during the last decades (Harrison, 1965; Tsay et al., 2004; Jang et al., 2002), this approach has found so far little attention for aluminium aerospace applications.

Therefore, the authors successfully gave the experimental proof of concept that it is possible to use this innovative approach for aluminium aircraft structures (Schnubel et al., 2012a) and that the resulting FCG can be predicted numerically using a finite element based prediction methodology (Schnubel and Huber, 2012a).

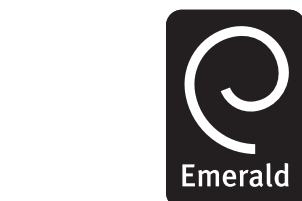

International Journal of Structural Integrity

Vol. 4 No. 4, 2013 pp. $429-445$ (C) Emerald Group Publishing Limited 1757-9864 DOI 10.1108/IJSI-08-2012-0020 
IJSI

4,4

430

In Sections 2 and 3 this work will be summarized to provide the theoretical basis for solving the design optimisation problem presented in Section 4. The capabilities as well as the current limitations of the proposed strategy for numerically based design optimisation are discussed for two specific cases, the optimum positioning of one and two heating lines on a C(T)100 specimen that leads to a maximised total lifetime.

\section{Laser heating process}

A Nd: YAG laser was used for the experiments to produce a line of laser heating on the surface of C(T)100 specimens made from the new aircraft alloy AA2198-T8 with a thickness of $5 \mathrm{~mm}$. The sheet thickness of $5 \mathrm{~mm}$ was selected to prevent buckling of the specimens during the FCG experiments. However, there are no problems expected to apply the laser heating process also to thinner or thicker sheet material. During the process, the laser travelled at a fixed heating line position $x_{\mathrm{h}}=55 \mathrm{~mm}$ and with a constant speed of $v_{\mathrm{y}}=3.33 \mathrm{~mm} / \mathrm{s}$ in positive y-direction from one edge of the specimen to the other. Since the goal was to apply the heating without local melting of the material, a welding optic was used, but the working distance was increased to obtain a laser spot diameter of approximately $5 \mathrm{~mm}$. A layer of silicon carbide powder with a thickness of $0.5 \mathrm{~mm}$ was placed on the irradiated surface to ensure an equal absorption of the laser light for all specimens.

Figure 1(a) shows a photograph of a specimen after laser heating with a schematic view of the resulting residual stress state (Schnubel et al., 2012a). After heating, tensile longitudinal residual stresses $\sigma_{\text {yy }}$ with a magnitude of $130 \mathrm{MPa}$ can be found in the heating area with balancing compressive residual stresses in the remaining part of the specimen with a nearly constant value of approximately $-30 \mathrm{MPa}$. This is qualitatively similar to the residual stress state after welding.

Figure 1(b) shows a macrograph of the laser heating area (Schnubel et al., 2012a). The depicted vertical structuring is due to the elongated pancake-like grain structure,

Figure 1.

(a) Laser heated C(T)100 specimen prepared for FCG testing and (b) cross-section macrograph after laser heating

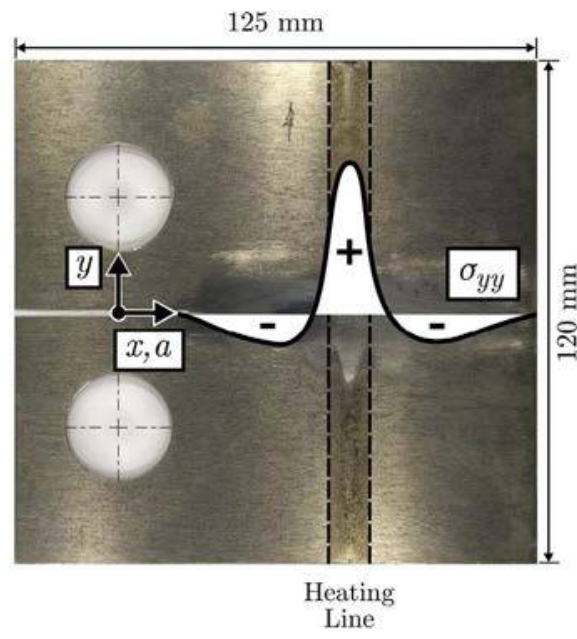

(a)

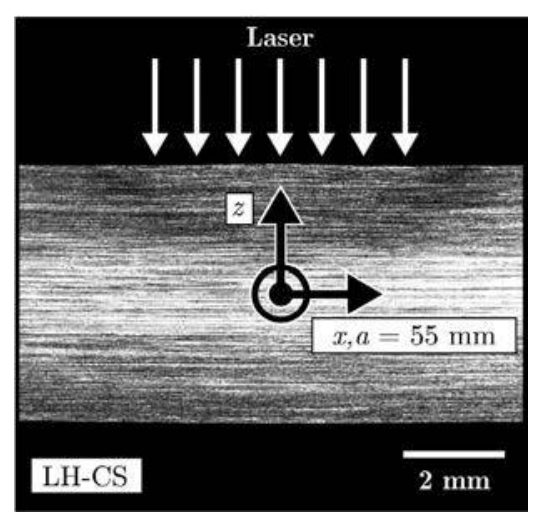

(b)

Source: Schnubel et al. (2012a) 
resulting from the thermo-mechanical treatment during the manufacturing. Only beneath the laser-irradiated surface area that is indicated by the white arrows, a slight colour change can be seen in a semi elliptical area. However, no signs of partial melting or surface crack formation were found.

While Figure 2(a) gives a contour plot of the measured microhardness after laser heating, Figure 2(b) shows the results for $z=1.8 \mathrm{~mm}$ as line plot (Schnubel et al., 2012a). A significant drop of the microhardness can be seen in the area beneath the laser irradiated surface. For orientation, the lateral extension of the heating zone (HZ) and the heat affected zone (HAZ) are marked light shaded. The remaining part of the specimen shows the hardness of the base material (BM).

Thermocouple measurements were performed to characterise the process temperature field. The heating induced residual stresses were measured on one specimen via synchrotron diffraction using the HARWI II beamline at DESY, Hamburg. Tests comparing the FCG of BM and laser heated specimens were performed under constant amplitude loading with $F_{\max }=4.41$ and $R=0.1$; these values are in a typical load range for damage tolerance tests of primary aluminium aircraft structural members with a increase of $\Delta \mathrm{K}_{\text {appl }}$ from approx. $10 \mathrm{MPa} \sqrt{\mathrm{m}}$ to $35 \mathrm{MPa} \sqrt{\mathrm{m}}$ with increasing crack length during the test. The results of these tests have been published in Schnubel et al. (2012a) and are shown in the next section in direct comparison to the numerical prediction results.

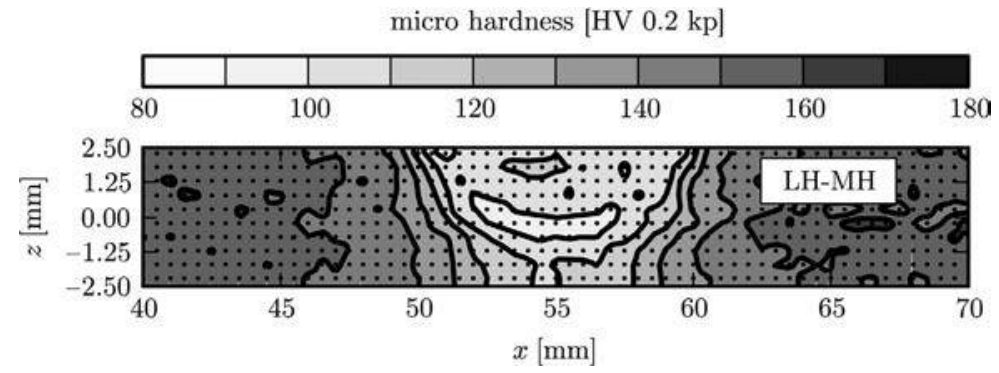

(a)

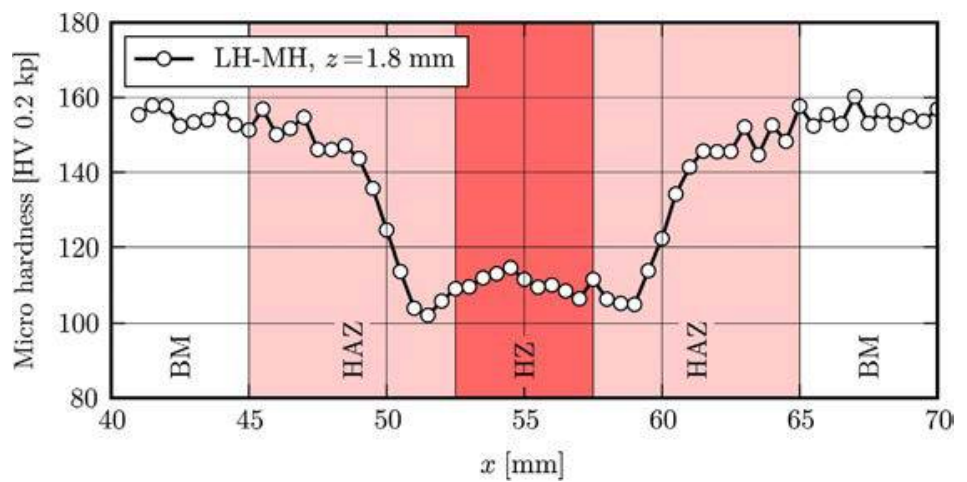

(b)

\author{
Retardation \\ of fatigue \\ crack growth
}

431 
IJSI

4,4

432

\section{Numerical prediction methodology}

The prediction of the FCG in structures and components containing residual stresses is a classical fracture mechanics problem. There are two main effects of residual stresses with regard to FCG (Schnubel et al., 2012a):

(1) If the crack faces stay completely open during the load cycle under combined loading with applied external load and residual stresses, the residual stresses influence $K_{\text {tot }}$ under minimum and maximum load in the same way through superposition of both stress fields. Accordingly, their only effect is a shift of the total stress intensity ratio $R_{\text {tot. }}$. For compressive residual stresses $R_{\text {tot }}<R_{\text {appl }}$ and for tensile residual stresses $R_{\text {tot }}>R_{\text {appl }}$. Since the crack growth rate is rather insensitive to the stress ratio, this leads only to a small effect compared to the residual stress free state.

(2) In the presence of compressive residual stresses, it is possible that the crack faces are closed also for loads larger than the minimum applied load but are completely open under maximum applied load. In this case $K_{\text {tot }}\left(F_{\max }\right)<K_{\text {appl }}\left(F_{\max }\right)$ and due to the closed crack faces at lower loads $K_{\text {tot }}\left(F_{\min }\right)=0 \mathrm{MPa} \sqrt{ } \mathrm{m}$. Hence, the total stress intensity factor range $\Delta K_{\text {tot }}$ is reduced and the total stress intensity factor ratio $R_{\text {tot }}=0$. Since the crack growth rate is very sensitive to the stress intensity factor range, this can create a huge retardation effect.

During the last decades the following methodology for the prediction the FCG in residual stress fields has found common acceptance (Parker, 1982; Terada, 2005; Edwards, 2006; Irving et al., 2009). In a first step, the residual stress intensity factor $K_{\text {res }}$ due to the internal residual stresses alone is calculated on basis of the measured residual stress component that acts perpendicular to the crack growth direction using for example the finite element method or the weight function method. Then, the total stress intensity $K_{\text {tot }}=K_{\text {res }}+K_{\text {appl }}$ is calculated as the sum of $K_{\text {res }}$ and the applied stress intensity $K_{\text {appl }}$ using the superposition law. Finally, the FCG rate $\mathrm{d} a / \mathrm{d} N$ is calculated by using $K_{\text {tot }}$ in an empirical crack growth law, such as the Walker equation.

In general, this approach shows good results. However, with regard to the presented laser heating of C(T)100 specimens there are two major limitations that need to be addressed. First, for cracks growing through compressive residual stress fields, nonlinear contact corrections are needed to ensure a physical sound description of the experimental conditions in the predictions (Parker, 1982). Introducing these contact corrections for preventing an unsound overlapping of the crack faces adds a nonlinearity. Hence, the assumption of linearity is violated and the superposition law is not valid any more. As a result an individual calculation of $K_{\text {tot }}$ needs to be performed for each individual load case accounting for the nonlinear dependencies. This aspect was discussed in detail elsewhere (Schnubel and Huber, 2012b).

Second, residual stress measurements are costly, difficult to perform and they normally only deliver specific components of the stress tensor. Additionally, there is a need to perform a large number of measurements, if for example a design parameter like the heating line position shall be examined.

Motivated by these points and adopting the idea presented by Jang et al. (2002) to use numerical predictions of heating induced residual stresses as basis for the following fracture mechanics analysis, the extended methodology shown in Figure 3 was developed, consisting of the following steps (Schnubel and Huber, 2012a): 


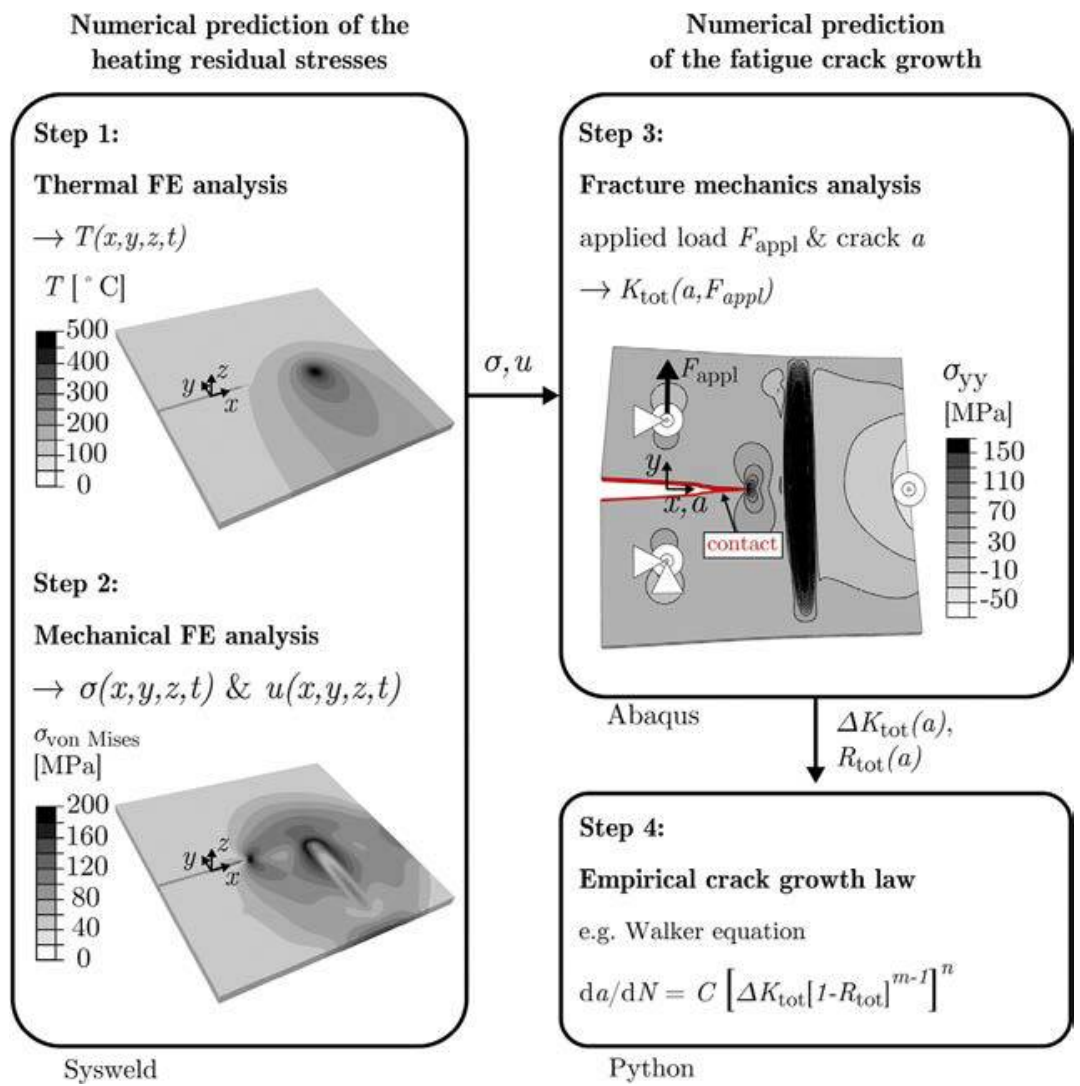

Source: Schnubel and Huber (2012a)
Retardation of fatigue crack growth

433

Figure 3.

Developed numerical prediction approach, taken from

(1) Use of a thermal $\mathrm{FE}$ analysis to predict the transient heating temperature field.

(2) Use of a mechanical FE analysis incorporating the thermal expansions and temperature dependent plasticity to predict the full residual stress field after heating.

(3) Extraction of the total stress intensity factor $K_{\text {tot }}$ in a fracture mechanics analysis using the modified virtual crack closure technique (Krueger, 2002). In this nonlinear analysis step the loaded model with the mapped predicted heating residual stresses and distortions is incrementally cut open and a contact condition is added continuously for the newly generated crack faces. Hence, no use of the superposition principle is made.

(4) Calculation of the FCG rate $\mathrm{d} a / \mathrm{d} N$ by using the extracted $K_{\text {tot }}$ in an empirical crack growth law.

A Goldak ellipsoid volume heat source (Radaj, 2003) was used in the thermal analysis for modelling the heat flux into the model. The first step in the thermal analysis is the iterative calibration of this heat source to match the real process conditions that were 
IJSI

4,4

434

Figure 4.

(a) Measured (EXP) and predicted (FEM) peak temperatures and (b) measured (EXP) and predicted (FEM) residual stresses characterized via thermocouple measurements for two specimens. Figure 4(a) shows the comparison of the measured and numerically predicted peak temperatures $T_{\max }$ during the thermo cycle as function of the $x$-position. As shown, an excellent agreement has been achieved with the calibrated heat source for the used process parameter set. Further details on the measurement results are given in Schnubel et al. (2012a). After this calibration the heat source can be used in Section 4 to study the effect of the heating line position without any need for further calibration. Details on the calibration procedure und model parameters were published in Schnubel and Huber (2012a).

Figure 4(b) shows the comparison of the measured and predicted residual stress difference $\Delta \sigma=\sigma_{\mathrm{yy}}-\sigma_{\mathrm{xx}}$. It can be stated that the mechanical simulation delivers a reasonable prediction for the residual stresses, even though higher values of $\Delta \sigma$ are predicted in the $\mathrm{HZ}$ compared to the measurements. In addition to the $\Delta \sigma$, the numerically predicted longitudinal stresses $\sigma_{\mathrm{yy}}$ (dotted) and transverse stresses $\sigma_{\mathrm{xx}}$ (dashed) are shown. The amplitude of $\sigma_{\mathrm{xx}}$ is rather small compared to the one of $\sigma_{\mathrm{yy}}$. Thus, $\Delta \sigma$ is dominated by the contribution of $\sigma_{\mathrm{yy}}$. The effect of the applied boundary conditions and material properties on the predicted residual stresses is discussed in Schnubel and Huber (2012a).

For the FE fracture mechanics analysis a separate simulation was defined for each crack length $a$, using the deformed mesh and the full stress field from the last time step of the process simulation after cooling to room temperature. In this mechanical restart simulations pure linear elastic material behaviour with a Young's modulus $E=73 \mathrm{GPa}$ and a Poisson's ratio $v=0.33$ was assumed. An aspect that has been addressed by some researchers in the past, e.g. in Edwards (2006), is the potential influence of the redistribution of the residual stresses during to the crack growth on the predictions. By the use of the presented $\mathrm{FE}$ fracture mechanics analysis including a contact definition for the crack faces such redistribution effects are included naturally in the simulations.

The nodal displacements directly behind the crack tip and nodal forces at the crack tip were extracted and used for the calculation of $K_{\mathrm{tot}}\left(F_{\mathrm{appl}}, a\right)$ as function of the applied load $F_{\text {appl }}$ and the crack length $a$ via the modified virtual crack closure technique. The results for $K_{\text {tot }}\left(F_{\max }=4.4 \mathrm{kN}\right.$, a $)$ and $K_{\text {tot }}\left(F_{\min }=0.4 \mathrm{kN}\right.$, a $)$ based on the predicted laser heating residual stresses are shown in Figure 5(a) (LH-FEM). For comparison, also the results calculated for a model without residual stresses are shown (BM-FEM) what corresponds to BM behaviour. The following sections give a summary of the discussion presented in Schnubel and Huber (2012a).

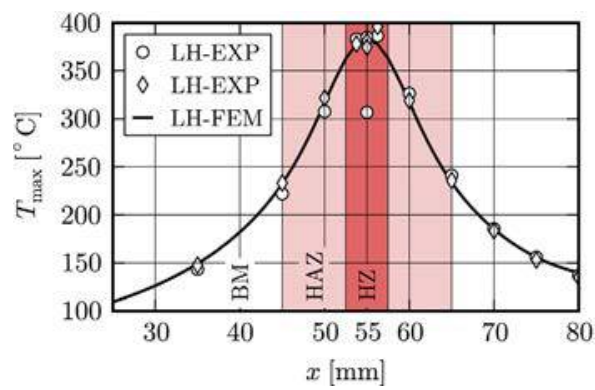

(a)

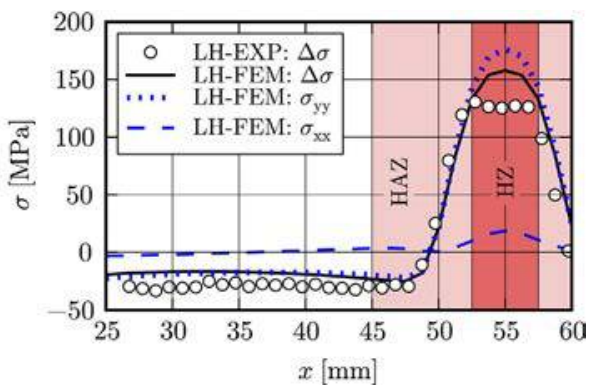

(b)
Source: Schnubel et al. (2012a) and Schnubel and Huber (2012a) 
For $a<49 \mathrm{~mm}$ the crack tip is situated in the area of compressive $\sigma_{\mathrm{yy}}$ for the case LH-FEM including the laser heating residual stresses shown in Figure 4(b). Hence, the $K_{\text {tot }}\left(F_{\text {max }}\right)$ is lowered in comparison to the simulations without residual stresses. Under minimum applied load the crack faces at the crack tip stay completely closed as indicated by $K_{\text {tot }}\left(\mathrm{F}_{\text {min }}\right)=0 \mathrm{MPa} \sqrt{\mathrm{m}}$. In conclusion, this results in a reduction of total stress intensity factor range $\Delta K_{\text {tot }}=K_{\text {tot }}\left(F_{\text {max }}\right)-K_{\text {tot }}\left(F_{\min }\right)$ for the case LH-FEM.

After the crack tip enters the area of tensile residual stresses $\sigma_{\mathrm{yy}}$, for $a>49 \mathrm{~mm}$, the values of $K_{\text {tot }}\left(F_{\min }\right)$ and $K_{\text {tot }}\left(F_{\max }\right)$ increase subsequently until both exceed the corresponding values from the simulations without residual stresses, i.e. the total stress intensity factor ratio $R_{\text {tot }}=K_{\text {tot }}\left(F_{\text {min }}\right) / K_{\text {tot }}\left(F_{\text {max }}\right)$ increases.

For the prediction of $\mathrm{d} a / \mathrm{d} N$, the calculated $K_{\text {tot }}$ were used in the Walker equation (Figure 3) with material constants of AA2198-T8 that were fitted on basis of BM FCG tests including three different R-ratios (Schnubel and Huber, 2012a). The predicted FCG rates for BM specimens (BM-FEM) and laser heated specimens (LH-FEM) are in good agreement with the experimental data (BM-EXP, LH-EXP), as seen from Figure 5(b).

For the BM specimens without residual stresses this is only a graphical verification that the used material constants of the Walker equation were correctly identified. Furthermore, the good agreement of the prediction and the measurement results for the laser heated specimens ( $\mathrm{LH}$ ) validates the developed approach of using the predicted laser heating induced residual stresses as basis for the prediction of the FCG.

\section{Simulation based design optimisation}

In this section, the predictive approach which has been summarized in the previous section is used to solve two design optimisation problems. In this context the previously published work deals with the predictive modelling for solving the direct problem in four steps according to Figure 3 while in the following a complex inverse problem shall be addressed. The goal is to find the optimum positioning of either one or two heating lines on a C(T)100 specimen that leads to a maximised total lifetime. Finally, the prediction results are validated experimentally for selected cases followed by a discussion on how to apply the proposed optimisation strategy to real components.

\subsection{Optimisation with one heating line}

Figure 6 shows the principle of the first design optimisation study on the positioning of one heating line. The goal of the optimisation was to identify the heating line position

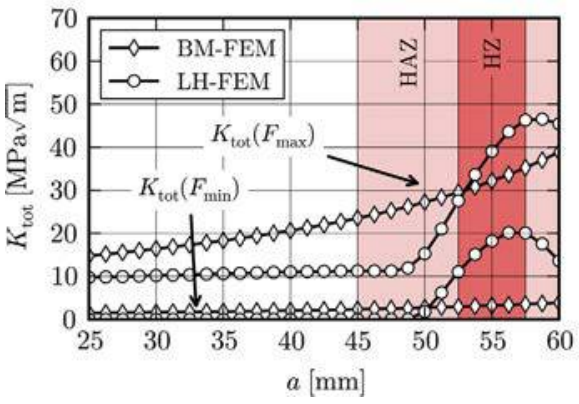

(a)

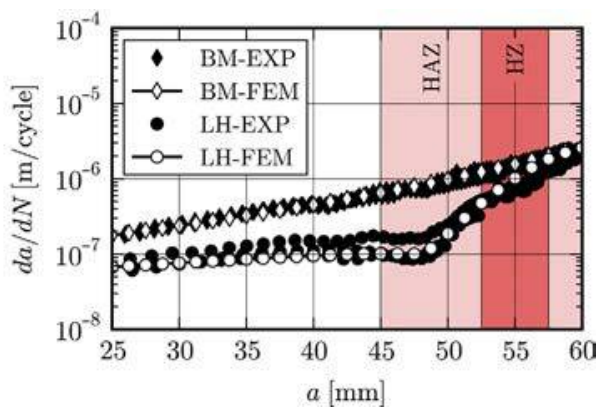

(b)
Source: Schnubel et al. (2012a) and Schnubel and Huber (2012a)

\section{Retardation of fatigue crack growth}

435
Figure 5.

(a) Predicted $K_{\text {tot }}$ and (b) measured and predicted da/dN for BM specimens (BM) as well as laser heated specimens (LH) 
IJSI

4,4

436

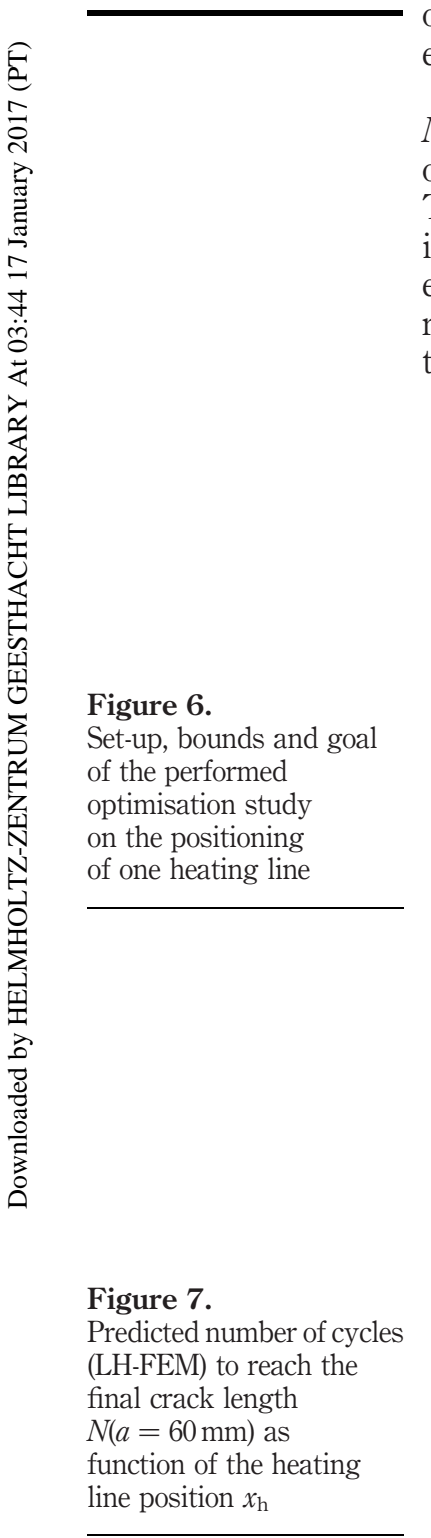

$x_{\text {h,opt }}$ leading to a maximum number of load cycles $N$ before a crack with an initial length $a_{0}=25 \mathrm{~mm}$ reaches a final crack length of $a=60 \mathrm{~mm}$.

The model used for the numerical design optimisation was adopted from the validation case presented in Sections 2 and 3, including the same boundary conditions and material properties. Only the heating line position $x_{\mathrm{h}}$ was varied in steps of $2.5 \mathrm{~mm}$ for $25 \mathrm{~mm} \leq x_{\mathrm{h}} \leq 85 \mathrm{~mm}$. Because the optimised heating positions induce a very effective retardation effect, the applied loads were increased to 150 per cent of the original values to facilitate and speed up the subsequently performed FCG experiments.

Figure 7 shows the predicted number of cycles to reach the final crack length $N(a=60 \mathrm{~mm})$ as function of the heating line position $x_{\mathrm{h}}$. For the given design optimisation problem, the optimum line position can be identified as $x_{\mathrm{h}, \mathrm{opt}}=45 \mathrm{~mm}$. The simulation results show that a substantial retardation effect can be reached, which is positive for all positions $x_{\mathrm{h}}$. There is no indication for an inversion of the retardation effect due to an unfavourable placement of the laser heating line. In the worst case the retardation effect approaches zero, i.e. the laser heated material behaves equivalent to the BM (see dashed line in Figure 7).
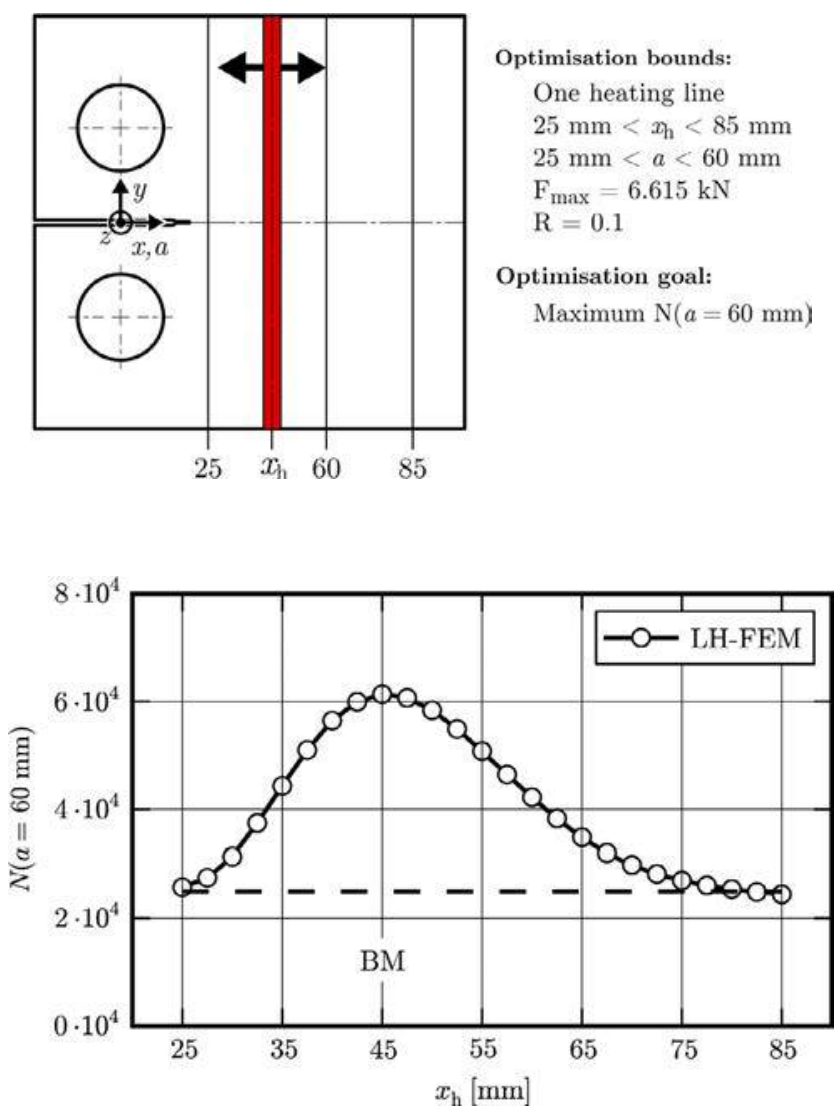
The predicted FCG behaviour was experimentally validated for the three heating line positions $x_{\mathrm{h}}=35 \mathrm{~mm}, x_{\mathrm{h}}=x_{\mathrm{h}, \mathrm{opt}}=45 \mathrm{~mm}$ and $x_{\mathrm{h}}=55 \mathrm{~mm}$. In a first attempt to perform these tests, an initial fatigue crack of the length $a=24 \mathrm{~mm}$ was introduced in the specimens prior to the application of the laser heating. However, this procedure did not prevent a formation of secondary cracks at the fixture holes during the tests, as expected, but further promoted that phenomenon. For each of the three heating line positions one specimen was tested and in each of this tests secondary cracks growing from the fixture holes where found, while the growth of the main crack was arrested.

In order to explain the observed behaviour, a further process simulation model was prepared including also a sharp initial fatigue crack. The simulation results suggested that the transient process stress fields were leading to the formation of a large plastic zone at the crack tip, i.e. a kind of overload effect. From fatigue experiments with varying amplitude loading it is well known that such formation of a plastic zone can significantly retard the FCG (Janssen et al., 2004; Schwalbe, 1980; Daneshpour et al., 2012).

To avoid further problems with secondary cracking from the fixture holes, for all remaining specimens the fatigue crack with the length $a=24 \mathrm{~mm}$ introduced prior to laser heating was removed together with the formed plastic zone by extending the initial notch length from $x=20 \mathrm{~mm}$ to $x=30 \mathrm{~mm}$ via machining and the FCG tests were completed.

The FCG rates $\mathrm{d} a / \mathrm{d} N$ calculated on basis of the optical crack length measurement data are shown in Figure 8 in comparison to the predictions. As discussed in (Schnubel, 2012b) the FCG in laser heated specimens is highly sensitive to the process temperature fields. Therefore, the observed scatter of the experimental data is most

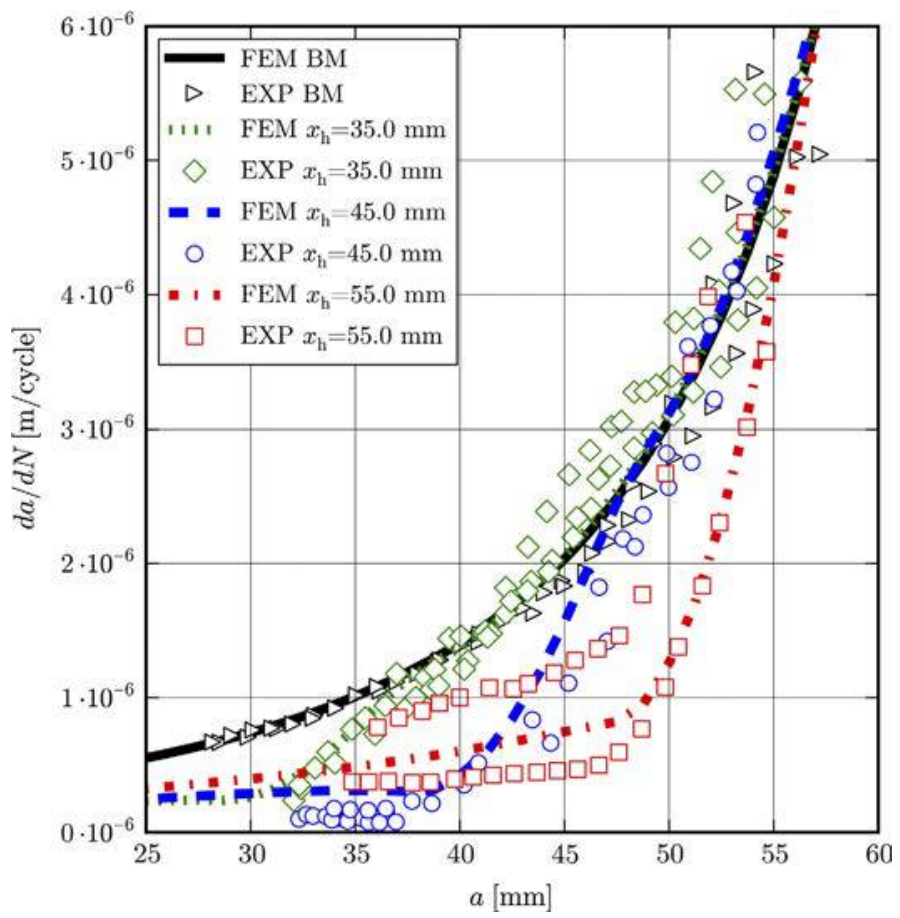

\section{Retardation of fatigue crack growth}

437
Figure 8.

Predicted and measured FCG rate $\mathrm{d} a / \mathrm{d} N$ as function of the crack length for three selected heating line positions $x_{\mathrm{h}}$ 
IJSI

4,4

438

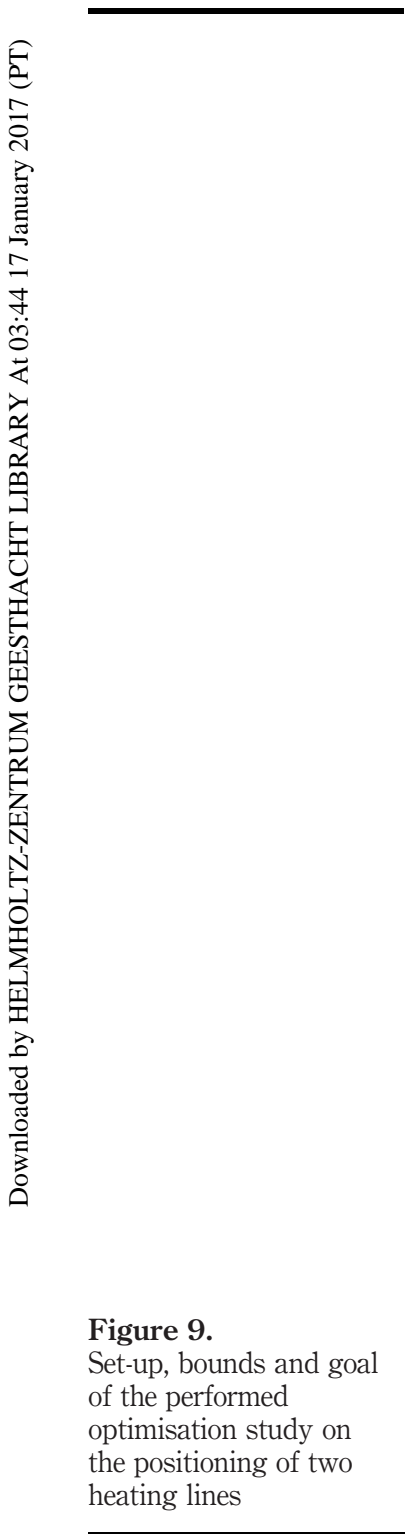

likely a direct result of small changes of the process conditions during the specimen manufacturing. Anyway, the predictions match the experimental results within the experimental scatter, indicating that the chosen optimisation approach provides adequate prediction for the optimum heating line position $x_{\mathrm{h}, \mathrm{opt}}$.

Figure 8 also shows two opposing effects. With a closer heating line position $x_{\mathrm{h}}$ relative to the initial position of the crack tip $a_{0}=25 \mathrm{~mm}$ the initial FCG is slower but also the period of slow crack growth is shorter. The crack growth is only retarded significantly until the crack tip reaches the corresponding position $x_{\mathrm{h}}$. In conclusion, the identified optimum position $x_{\mathrm{h} \text {,opt }}$ provides the best balance between these two opposing effects for the chosen optimisation bounds.

\subsection{Optimisation with two heating lines}

Figure 9 shows the principle of the second design optimisation study on the positioning of two heating lines. In the process simulations the application of two heating lines was executed by running a first analysis creating a heating line at $x_{\mathrm{h} 1}$ that was followed by a second restart simulation creating a heating line at $x_{\mathrm{h} 2}$ while using the simulation results of the first run as initial values. The remaining model parameters for the process simulation like the clamping conditions or the material properties remained unchanged.

As seen from the microhardness measurements shown in Figure 2, laser heating leads to a change of the local mechanical properties in the $\mathrm{HZ}$ and the HAZ. This implies that also the temperature dependent yield stress $\sigma_{\mathrm{ys}}(T)$ is locally changed in the HZ and HAZ. On the one hand, the heating induced residual stresses are highly dependent on $\sigma_{\mathrm{ys}}(T)$ in the heating area as discussed in (Schnubel, 2012b). On the other hand, the used process simulation does not capture the local changes of $\sigma_{\mathrm{ys}}(T)$ after the application of a first heating line. Hence, for cases in which the second heating line is placed on top of the first one, the process simulation does not deliver correct predictions for the final residual stress state. For this reason the optimisation bounds shown in Figure 9 specifically exclude the designs with $x_{\mathrm{h} 1}=x_{\mathrm{h} 2}$. Even though there exist models to account for the changes of the precipitation state during welding or heating simulation (Myhr and Grong, 1991a, b) the needed model calibration for a specific material is connected with a very high experimental effort, when this material is not yet included in a commercial database, such as that of SYSWELD. This is the case for AA2198-T8 used in this work. The remaining optimisation bounds are chosen similar as for the previous example with only one heating line. The positions of the two heating lines were allowed to change independently within the interval $25 \mathrm{~mm}<x_{\mathrm{h} 1}, x_{\mathrm{h} 2}<85 \mathrm{~mm}$ and the crack growth for

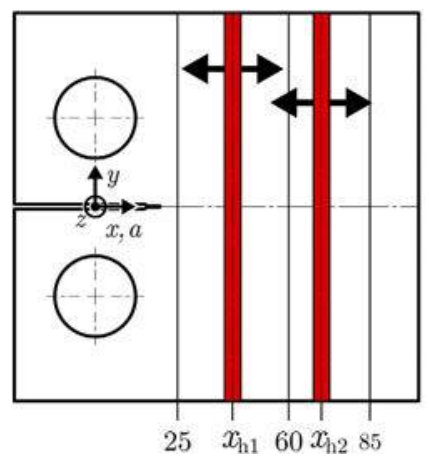

Optimisation bounds:

Two heating lines

$25 \mathrm{~mm}<x_{\mathrm{h} 1}, x_{\mathrm{h} 2}<85 \mathrm{~mm}$

$x_{\mathrm{h} 1} \neq x_{\mathrm{h} 2}$

$25 \mathrm{~mm}<a<60 \mathrm{~mm}$

$\mathrm{F}_{\max }=6.615 \mathrm{kN}$

$\mathrm{R}=0.1$

Optimisation goal:

Maximum $\mathrm{N}(a=60 \mathrm{~mm})$ 
$25 \mathrm{~mm}<a<60 \mathrm{~mm}$ was considered. The optimisation goal was the identification of the optimum heating line position combination $x_{\mathrm{h} 1, \mathrm{opt}}$ and $x_{\mathrm{h} 2, \mathrm{opt}}$ resulting in a maximum number of cycles $N$ until reaching the final crack length $a=60 \mathrm{~mm}$.

Due to the increased number of possible combinations of $x_{\mathrm{h} 1}$ and $x_{\mathrm{h} 2}$ compared to the positioning of only one line, a coarser grid for the discretely simulated combinations with a step width of $10 \mathrm{~mm}$ was chosen. Excluding the combinations with $x_{\mathrm{h} 1}=x_{\mathrm{h} 2} \mathrm{a}$ total of 42 simulations had to be performed to calculate all allowed combinations of the heating line positions using the given grid step width within the given bounds. The simulation results for one heating line showed that substantial plastic yielding with equivalent plastic strains of more than 0.5 per cent during heating is limited to a narrow area of approximately $3.75 \mathrm{~mm}$ to each side of the heating line centre. The microhardness measurements (Figure 2) show large relative changes of the material properties of more than 10 per cent, which are limited to a zone of approximately $6 \mathrm{~mm}$ to each side of the heating line centre. Hence, as long as the heating lines have a minimum distance of $10 \mathrm{~mm}$, the locally altered material properties are not supposed to have a large effect on the predicted residual stresses and a possible coupling or history effect can be neglected.

Figure 10 shows the predicted $N(a=60 \mathrm{~mm})$ as function of $x_{\mathrm{h} 1}$ and $x_{\mathrm{h} 2}$. The contour plot was generated using the natural neighbour interpolation on basis of the

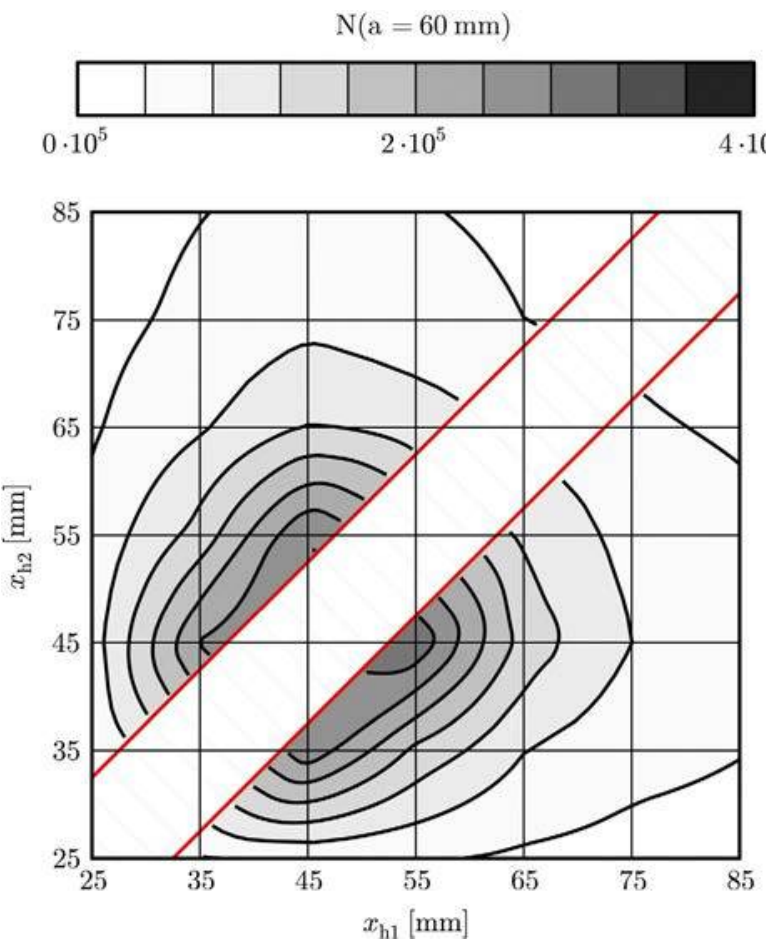

Notes: The red hatched parameter area was excluded from the simulations, since there underlying assumptions of the process simulation are violated

\section{Retardation of fatigue crack growth}

439
Figure 10.

Predicted number of cycles until reaching the final crack length $N(a=60 \mathrm{~mm})$ as function of the heating line positions $x_{\mathrm{h} 1}$ and $x_{\mathrm{h} 2}$ 
IJSI

4,4

440

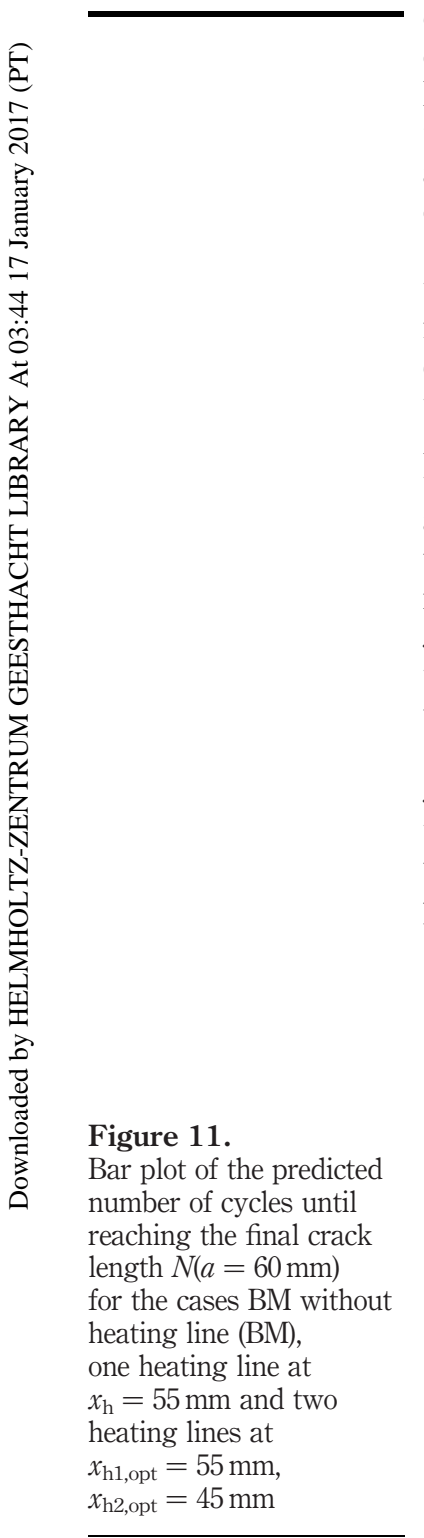

42 specifically simulated positioning combinations. The parameter region excluded from the predictions, with $x_{\mathrm{h} 1}=x_{\mathrm{h} 2}$, is marked red and hatched. The results are not exactly symmetric with regard of the sequence of the line positioning. For example, the predicted $N(a=60 \mathrm{~mm})$ for $x_{\mathrm{h} 1}=55 \mathrm{~mm}, x_{\mathrm{h} 2}=45 \mathrm{~mm}$ is larger than for $x_{\mathrm{h} 1}=45 \mathrm{~mm}$, $x_{\mathrm{h} 2}=55 \mathrm{~mm}$. The optimum configuration can be identified as the combination $x_{\mathrm{h} 1, \mathrm{opt}}=55 \mathrm{~mm}$ and $x_{\mathrm{h} 2, \mathrm{opt}}=45 \mathrm{~mm}$.

In Figure 11 a comparison of the predicted $N(a=60 \mathrm{~mm})$ is given for the BM, the configuration containing only one heating line at $x_{\mathrm{h}}=x_{\mathrm{h} 1, \mathrm{opt}}=55 \mathrm{~mm}$ and the identified optimum configuration for two heating lines $x_{\mathrm{h} 1, \mathrm{opt}}=55 \mathrm{~mm}, x_{\mathrm{h} 2, \mathrm{opt}}=45 \mathrm{~mm}$. By applying one heating line at $x_{\mathrm{h}}=x_{\mathrm{h} 1, \mathrm{opt}}=55 \mathrm{~mm}$, the total predicted lifetime is increased by approximately 100 per cent compared to the BM behaviour. The addition of a second heating line at $x_{\mathrm{h} 2, \mathrm{opt}}=45 \mathrm{~mm}$ leads to an increase of the predicted total lifetime of approximately 900 per cent compared to the BM behaviour.

For the identified optimum configuration, with $x_{\mathrm{h} 1, \mathrm{opt}}=55 \mathrm{~mm}$ and $x_{\mathrm{h} 2, \mathrm{opt}}=45 \mathrm{~mm}$, the predictions have been validated experimentally. The specimens for these tests were manufactured along with the ones used in the previous section for the validation experiments with only one heating line. A fatigue crack of $a=24 \mathrm{~mm}$ was induced prior to the laser heating. Then, the first laser heating line was the applied at $x_{\mathrm{h} 1, \mathrm{opt}}=55 \mathrm{~mm}$. After the specimens cooled down to room temperature, the silicon carbide powder from the first run was removed and the specimen surface was manually cleaned prior to the application of the second heating line at $x_{\mathrm{h} 2, \mathrm{opt}}=45 \mathrm{~mm}$. The clamping of the specimens was removed after the application of the second heating line and the following cooling to room temperature. For this set of specimens the initial notch was also extended from $x=20 \mathrm{~mm}$ to $x=30 \mathrm{~mm}$ after the laser heating to remove the initial fatigue crack and the process induced plastic zone in front of the crack tip. Then, testing was performed using the same conditions as described earlier.

Figure 12 shows the predicted FCG rate for the optimum configuration, with $x_{\mathrm{h} 1, \mathrm{opt}}=55 \mathrm{~mm}$ and $x_{\mathrm{h} 2, \mathrm{opt}}=45 \mathrm{~mm}$, together with the experimental results based on the optical crack length measurement data the predictions match the experiments within the experimental scatter, indicating that the chosen optimisation approach provides correct predictions also for this case.

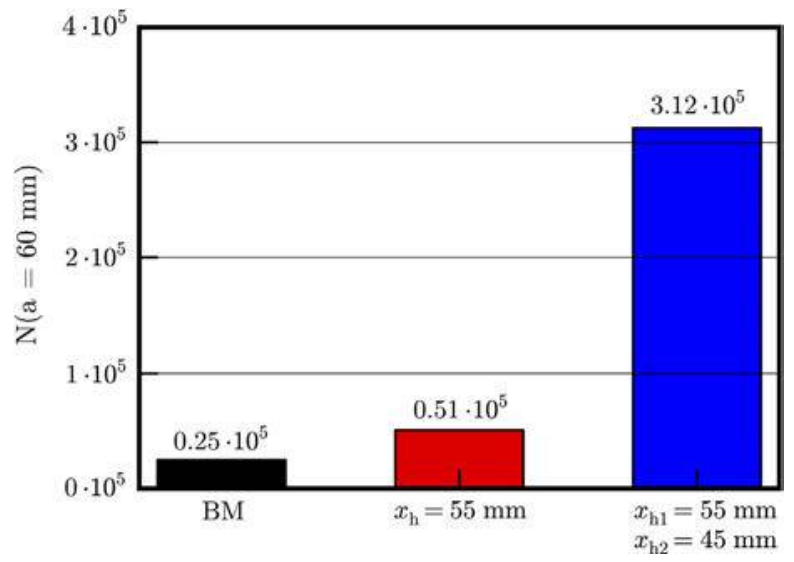




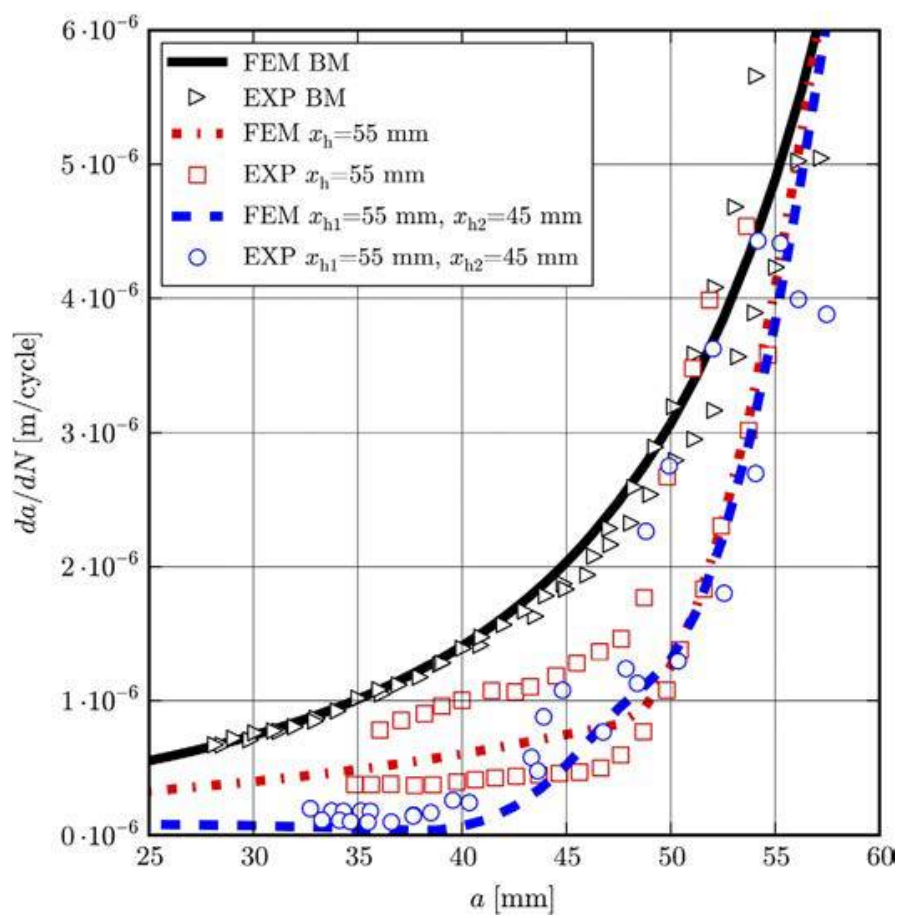

\section{Retardation of fatigue crack growth}

441

Figure 12.

Predicted and measured

FCG rate for the cases

$\mathrm{BM}$ without heating

line $(\mathrm{BM})$, one heating

line at $x_{\mathrm{h}}=55 \mathrm{~mm}$ and two heating lines at $x_{\mathrm{h} 1 \text {,opt }}$ $=55 \mathrm{~mm}, x_{\mathrm{h} 2 \text {,opt }}=45 \mathrm{~mm}$

For comparison, in Figure 12 also the results for the BM and the configuration containing only one heating line at $x_{\mathrm{h}}=x_{\mathrm{h} 1, \mathrm{opt}}=55 \mathrm{~mm}$ are shown. The addition of the second heating line at $x_{\mathrm{h} 2, \mathrm{opt}}=45 \mathrm{~mm}$ leads to a drop of the initial crack growth rate compared to the configuration with only one heating line at $x_{\mathrm{h}}=x_{\mathrm{h} 1, \mathrm{opt}}=55 \mathrm{~mm}$. However, after reaching the position $x_{\mathrm{h} 2, \mathrm{opt}}=45 \mathrm{~mm}$ the crack growth rate does not directly increase as it is the case for the specimens containing only one heating line at this position (Figure $8, x_{\mathrm{h}}=45 \mathrm{~mm}$ ). Instead, the crack growth is further retarded and shows a similar behaviour as the specimen containing only one heating line at $x_{\mathrm{h}}=55 \mathrm{~mm}$.

Figure 13 shows the predicted $\sigma_{\mathrm{yy}}, \Delta \mathrm{K}_{\mathrm{tot}}$ and $\mathrm{R}_{\mathrm{tot}}$ for the cases BM without heating line (BM), one heating line at $x_{h}=55 \mathrm{~mm}$ and two heating lines at $x_{h 1, o p t}=55 \mathrm{~mm}$, $\mathrm{x}_{\mathrm{h} 2, \mathrm{opt}}=45 \mathrm{~mm}$. For the configuration with two heating lines the compressive residual stresses are approximately twice as high as for the configuration with one heating line. This higher compressive residual stresses explain the large drop of the initial crack growth rate for $x<40 \mathrm{~mm}$ (Figure 12).

As seen from Figures 12 and $13\left(\sigma_{\mathrm{yy}}\right.$, top) for the case with two heating lines, the FCG is even further retarded compared to a single heating line, when the crack tip entered the area with longitudinal tensile residual stresses for $x>40 \mathrm{~mm}$. Even though $\mathrm{R}_{\text {tot }}$ becomes increasingly positive (Figure 13) indicating a complete opening of the crack faces at the crack tip under minimum applied load, $\Delta \mathrm{K}_{\text {tot }}$ is still lower compared to the reference configuration without residual stresses. This complex behaviour is due to partial opening of the crack faces under applied loading. An explanation of the underlying mechanisms and a discussion of their consequences on the FCG behaviour are given elsewhere (Schnubel and Huber, 2012b). 
IJSI

4,4

\section{2}
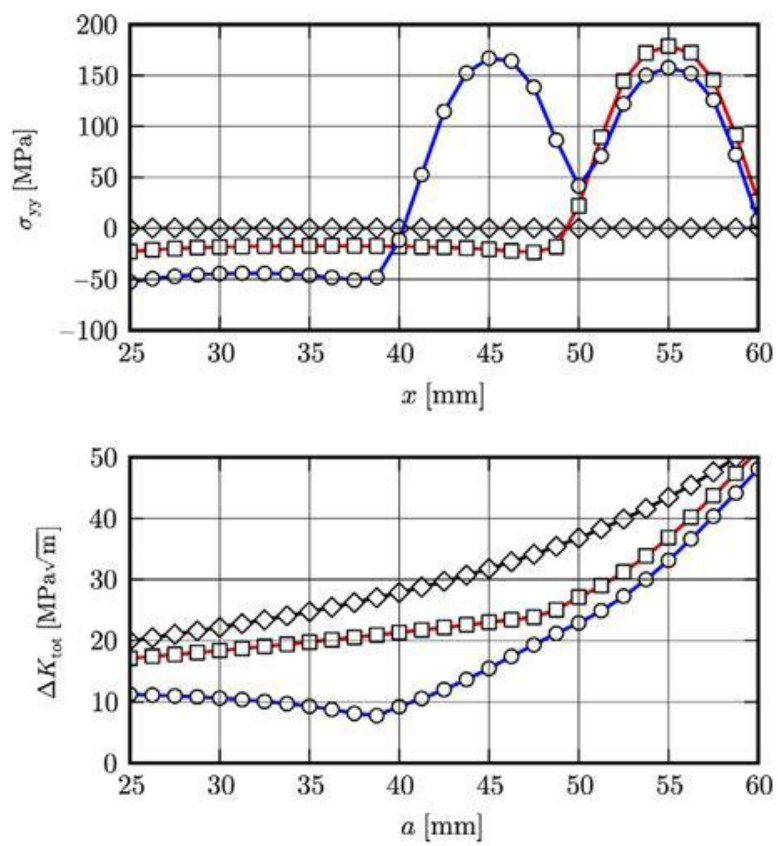

Figure 13.

Predicted longitudinal residual stresses $\sigma_{\mathrm{yy}}$, $\Delta \mathrm{K}_{\text {tot }}$ and $\mathrm{R}_{\text {tot }}$ for the cases $\mathrm{BM}$ without heating line (BM, diamonds), one heating line at $\mathrm{x}_{\mathrm{h}}=55 \mathrm{~mm}$ (boxes) and two heating lines at $\mathrm{x}_{\mathrm{h} 1, \mathrm{opt}}=55 \mathrm{~mm}$, $\mathrm{x}_{\mathrm{h} 2, \mathrm{opt}}=45 \mathrm{~mm}$ (circles)

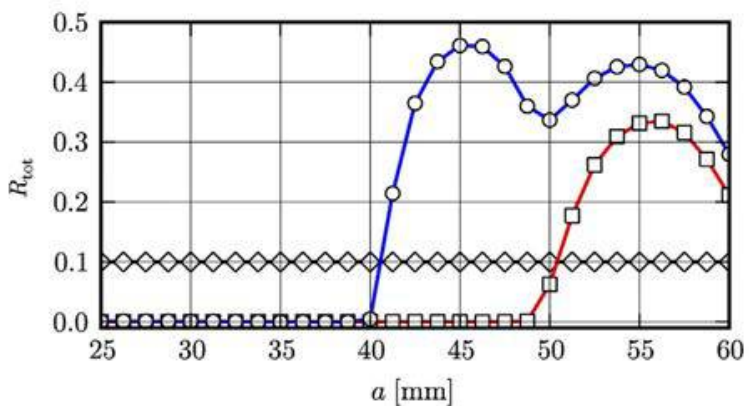

In conclusion of the achieved results presented in this section, the significantly increased total lifetime $N(a=60 \mathrm{~mm})$ for the optimum configuration, i.e. $x_{\mathrm{h} 1, \mathrm{opt}}=55 \mathrm{~mm}$ and $x_{\mathrm{h} 2, \mathrm{opt}}=45 \mathrm{~mm}$, is a result of a significant decrease of the initial crack growth rate as well as a longer preservation of the retardation effect in comparison to the residual stress designs obtained from only one heating line.

\subsection{Transferability to engineering structures}

It was shown in the previous sections that the presented FE-based optimisation approach can be used to study different design variants and to identify design parameters for achieving an optimum fatigue life. The identified optimum depends on the definition of the optimisation bounds, the starting crack length, the magnitude of applied load and the critical crack length for which the fatigue life shall be maximised. 
Particularly the presented case with two heating lines demonstrates how the proposed approach can be used for saving experimental costs. Using a pure experimental approach to identify the optimum configuration would have required manufacturing and testing of approximately 80 specimens (two per configuration) to cover the full variation range of only two parameters, which are the two heating line positions. The effort for studying a large number of parameter combinations is comparatively low using the predictive approach. Hence, the most promising designs can be identified computationally before manufacturing and testing. For the presented case of two heating lines only two specimens have been manufactured and tested for validating the identified optimum. This reduced the experimental effort by 97.5 per cent in relation to a pure experimental approach that would have been carried out with the same demand of quality as the computer optimisation.

Moreover, the costs on the scale of real components are that high that even in large coordinated projects only a few demonstrators can be manufactured and tested. Here the systematic application of predictive modelling tools for the optimisation preliminary to building and testing of a demonstrator is an essential asset. However, translation of the proposed principle of fatigue life enhancement via laser heating from lab scale to real engineering structures is also challenging. A prior structural analysis is needed to identify the relevant failure modes and locations and to define application relevant optimisation bounds and goals. This will lead to a set of conditions that has to be satisfied simultaneously by the optimisation approach.

As the next step towards this goal, our current work aims at up-scaling of the presented approach to large panels with five stringers. The task that needs to be solved first is the reduction of the computation time for large models for both, the process simulation as well as the crack propagation simulation.

\section{Conclusions}

A brief summary of the previously conducted work on laser heating including the development and validation of a finite element based prediction methodology for the FCG in laser heated specimens was presented. Then this prediction methodology coupling process simulation and subsequent fracture mechanics analysis was used to solve two design optimisation problems, the optimum placing of either one or two heating lines on a C(T)100 specimen in order to gain a maximum FCG life time.

In a first step, predictions for a set of variations of the heating line positions within the optimisation bounds were performed. Based on this prediction results the optimum line positions were identified. To show that this optimisation approach really delivers the correct optimum positions a final series of FCG experiments was performed. For all tested configurations the predictions matched the experiments within the experimental scatter indicating that the optimum heating line positions were identified correctly.

An interesting finding was that the observed maximum of the FCG life for one heating line originates from the best balance of two opposing effects. The initial crack growth is the slower if the heating line is placed closer to the initial crack tip, but on the other hand, the FCG is only retarded significantly until the crack tip reaches the corresponding heating line position. However, there is no indication of an inversion of the retardation effect due to an unfavourable positioning of the heating line. This underlines that the identified optimum positions are highly dependent on the specific optimisation bounds. For an application to real structures a prior analysis

\section{Retardation of fatigue crack growth}

443 
IJSI

4,4

444

step is needed to identify all relevant load cases and failure scenarios. Then these findings have to be translated to multiple constraints which simultaneously have to be considered for the optimisation.

With regard to the case with two heating lines the optimisation bounds had to exclude specifically the position combinations resulting in two lines placed on top of each other due to the existing limitations of the used process simulation. Within the defined bounds the optimum heating line positions were correctly identified. It can

be stated that the addition of a second heating line was leading to a large increase of the FCG life in the range of one order of magnitude constituting an impressive example of the potential for life enhancement provided by the laser heating technique.

In conclusion, it is demonstrated on the laboratory scale that laser heating has the potential to be employed for FCG retardation in aircraft aluminium structures and that in future the experimental effort needed for design optimisation can be significantly reduced using the developed numerically based approach.

\section{References}

Ball, D.L. and Lowry, D.R. (1998), "Experimental investigation on the effects of cold expansion of fastener holes", Fatigue \& Fracture of Engineering Materials \& Structures, Vol. 21 No. 1, pp. 17-34.

Daneshpour, S., Dyck, J., Ventzke, V. and Huber, N. (2012), “Crack retardation mechanism due to overload in BM and laser welds of Al alloys", International Journal of Fatigue, Vol. 42, pp. 95-103.

Edwards, L. (2006), "Influence of residual stress redistribution on fatigue crack growth and damage tolerant design”, Materials Science Forum, Vol. 524-525, pp. 77-82.

Harrison, J. (1965), "Exploratory fatigue tests on local heating as repair technique", British Welding Journal, Vol. 12, pp. 258-260.

Irving, P., Ma, Y.E., Zhang, X., Servetti, G., Williams, S., Moore, G., dos Santos, J. and Pacchione, M. (2009), "Control of crack growth rates and crack trajectories for enhanced fail safety and damage tolerance in welded structures", Proceedings of the 25th ICAF Sumposium of the International Committee on Aeronautical Fatigue Held in Rotterdam. The Netherlands, pp. 387-405.

Jang, C.D., Song, H.C. and Lee, C.H. (2002), "Fatigue life extension of a through-thickness crack using local heating", Proceedings of the Twelfth (2002) International Offshore and Polar Engineering Conference Held in Kitakyushu, Japan, pp. 224-229.

Janssen, M., Zuidema, J. and Wanhill, R. (2004), Fracture Mechanics, Spon Press, New York, NY.

Krueger, R. (2002), The Virtual Crack Closure Technique: History, Approach and Applications, NASA/CR-2002-211628, ICASE Report No. 2002-10, NASA Langley Research Center, Hampton, VA.

McClung, R.C. (2007), "A literature survey on the stability and significance of residual stresses during fatigue", Fatigue \& Fracture of Engineering Materials \& Structures, Vol. 30 No. 3, pp. 173-205.

Myhr, O. and Grong, Ø. (1991a), "Process modelling applied to 6082-T6 aluminium weldments I: reaction kinetics”, Acta Metallurgica et Materialia, Vol. 39, pp. 2693-2702.

Myhr, O. and Grong, Ø. (1991b), "Process modelling applied to 6082-T6 aluminium weldments II: applications of model", Acta Metallurgica et Materialia, Vol. 39, pp. 2703-2708. 
Parker, A.P. (1982), Stress Intensity Factors, Crack Profiles and Fatigue Crack Growth Rates in R stress Fields, Residual Stress Effects in Fatigue. ASTM STP 776, American Society for Testing and Materials, West Conshohocken, PA, pp. 13-31.

Radaj, D. (2003), Welding Residual Stresses and Distortion: Calculation and Measurement, DVS Verlag, Düsseldorf.

Reid, L. (2003), "Sustaining an aging aircraft fleet with practical life enhancement methods", paper presented at the RTO AVT Specialists' Meeting on "Life Management Techniques for Ageing Air Vehicles", Held in Manchester, UK, 8-11 October 2001, and Published in RTO Meeting Proceedings 79(II), NATO/RTO, pp. 32-1-32-12.

Schnubel, D. and Huber, N. (2012a), "Retardation of fatigue crack growth in aircraft aluminium alloys via laser heating - numerical prediction of fatigue crack growth", Computational Materials Science, Vol. 65, pp. 461-469.

Schnubel, D. and Huber, N. (2012b), "The influence of crack face contact on the prediction of fatigue crack propagation in residual stress fields", Engineering Fracture Mechanics, Vol. 84, pp. 15-24.

Schnubel, D., Horstmann, M., Ventzke, V., Riekehr, S., Staron, P., Fischer, T. and Huber, N. (2012), "Retardation of fatigue crack growth in aircraft aluminium alloys via laser heating experimental proof of concept", Materials Science and Engineering A, Vol. 546, pp. 8-14.

Schwalbe, K.H. (1980), Bruchmechanik Metallischer Werkstoffe, Carl Hanser Verlag, München.

Terada, H. (2005), "Stress intensity factor analysis and fatigue behavior of a crack in the residual stress field of welding", Iournal of ASTM International, Vol. 2, pp. 1-11.

Tsay, L.W., Young, M.C., Chou, F.Y. and Shiue, R.K. (2004), "The effect of residual thermal stresses on the fatigue crack growth of laser-annealed 304 stainless steels", Materials Chemistrv and Phvsics, Vol. 88, pp. 348-352.

\section{Corresponding author}

Dirk Schnubel can be contacted at: dirk.schnubel@hzg.de
To purchase reprints of this article please e-mail: reprints@emeraldinsight.com Or visit our web site for further details: www.emeraldinsight.com/reprints

\section{Retardation of fatigue crack growth}

445 
This article has been cited by:

1. Anne Groth, Manfred Horstmann, Nikolai Kashaev, Norbert Huber. 2015. Design of Local Heat Treatment for Crack Retardation in Aluminium Alloys. Procedia Engineering 114, 271-276. [CrossRef]

2. Daniel F.O. Braga, S.M.O. Tavares, Lucas F.M. da Silva, P.M.G.P. Moreira, Paulo M.S.T. de Castro. 2014. Advanced design for lightweight structures: Review and prospects. Progress in Aerospace Sciences 69, 29-39. [CrossRef] 